\title{
A New Mathematical Inventory Model with Stochastic and Fuzzy Deterioration Rate under Inflation
}

\author{
Bahar Naserabadi, Abolfazl Mirzazadeh, and Sara Nodoust \\ Department of Industrial Engineering, University of Kharazmi, Tehran, Iran \\ Correspondence should be addressed to Sara Nodoust; sara_arezoo_n@yahoo.com
}

Received 12 February 2014; Revised 19 June 2014; Accepted 8 July 2014; Published 14 August 2014

Academic Editor: Jiuping Xu

Copyright (C) 2014 Bahar Naserabadi et al. This is an open access article distributed under the Creative Commons Attribution License, which permits unrestricted use, distribution, and reproduction in any medium, provided the original work is properly cited.

\begin{abstract}
This paper develops an inventory model for items with uncertain deterioration rate, time-dependent demand rate with nonincreasing function, and allowable shortage under fuzzy inflationary situation. The goods are not deteriorating upon reception, but the deteriorating starts after elapsing a specified time. The lead time and inflation rate are both uncertain in the model. The resultant effect of inflation and time value of money is assumed to be fuzzy in nature and also we consider lead time as a fuzzy function of order quantity. Furthermore the following different deterioration rates have been considered: for the first case we consider fuzzy deterioration rate and for the second case we assume that the deterioration rate is time dependent and follows Weibull distribution with three known parameters. Since the inflation rate, deterioration rate, and the lead time are fuzzy numbers, the objective function becomes fuzzy. Therefore the estimate of total costs for each case is derived using signed distance technique for defuzzification. The optimal replenishment policy for the model is to minimize the total present value of inventory system costs, derived for both the above mentioned policies. Numerical examples are then presented to illustrate how the proposed model is applied.
\end{abstract}

\section{Introduction}

Many of the physical goods undergo decay or deterioration over time. Commodities such as fruits, vegetables, and foodstuffs are subject to direct spoilage during storage period. The highly volatile liquids such as gasoline, alcohol, and turpentine undergo physical depletion over time through the process of evaporation. The electronic goods, radioactive substances, photographic film, and grain deteriorate through a gradual loss of potential or utility with passage of time. The deteriorated items repairing is a major problem in the supply chain of most of the business organizations.

The first attempt to describe optimal ordering policies for deteriorating items was made by Ghare and Schrader [1]. Later, Covert and Philip [2] derived the model with variable deteriorating rate of two-parameter Weibull distribution.

Inventoried goods can be broadly classified into four metacategories:
(1) obsolescence which refers to items that lose their value through time due to rapid changes of technology or the introduction of a new product by a competitor;

(2) deterioration which refers to the damage, spoilage, dryness, vaporization, and so forth of the products;

(3) amelioration which refers to items whose value or utility or quantity increases with time;

(4) no obsolescence/deterioration/amelioration.

If the rate of obsolescence, deterioration, or amelioration is not sufficiently low, its impact on modelling of such an inventory system cannot be ignored. There are a few papers for obsolescing and ameliorating items. Moon et al. [3] considered the ameliorating/deteriorating items on an inventory model with time-varying demand pattern. Against obsolescing and ameliorating items, the deteriorating inventory models under inflationary conditions are studied greatly. 
In a few of these works, deterioration rate is not constant. For example, Chen [4] proposed an inflationary model with time proportional demand and Weibull distribution for deteriorating items using dynamic programming. Sana [5] proposed a model which deals with a stochastic economic order quantity (EOQ) model over a finite time horizon where uniform demand over the replenishment period is price dependent. The selling price is assumed to be a random variable that follows a probability density function. Moreover, the article suggests a new function regarding price-dependent demand. Sana [6] also presented another EOQ model over an infinite time horizon for perishable items where demand is price dependent and partial backorder is permitted. Based on the partial backlogging and lost sale cases, the author develops the criterion for the optimal solution for the replenishment schedule and proves that the optimal ordering policy is unique. Balkhi [7] presented a production lot-size inventory model where the production, demand, and deterioration rates are known, continuous, and differentiable functions of time. Shortages are allowed, but only a fraction of the stockout is backordered, and the rest is lost. De and Sana [8] developed a research that deals with a backorder EOQ model with promotional index for fuzzy decision variables. Here, a profit function is developed, where the function itself is the function of $m$ th power of promotional index (PI) and the order quantity; the shortage quantity and the PI are the decision variables. The demand rate is operationally related to PI variables and the model has been split into two types for the multiplication and addition operations. Lo et al. [9] introduced an integrated production-inventory model with imperfect production processes and Weibull distribution deterioration under inflation from the perspectives of both the manufacturer and the retailer. Sana [10] developed an EOQ model to determine the retailer's optimal order quantity for similar products. It is assumed that the amount of display space is limited and the demand of the products depends on the display stock level and the initiatives of sales staff where more stock of one product makes a negative impression of the other product. Roy Chowdhury et al. [11] presented an inventory model for perishable items with stock and advertisement sensitive demand. Singh and Pattnayak [12] developed a two-warehouse inventory model for deteriorating items with linear demand under conditionally permissible delay in payment. Sivashankari and Panayappan [13] studied a production-inventory model with reworking of imperfect production, scrap, and shortages.

Effect of inflation and time value of money in inventory problems is well established. The initial attempt in this direction was made by Buzacott [14]. He dealt with an EOQ model under inflation subject to different types of pricing policies. Soleimani-Amiri et al. [15] compared two methods for calculating the optimal total cost in an inventory model with time value of money and inflation for deteriorating items. Hou [16] developed an inflation model for deteriorating items with stock-dependent consumption rate and completely backordered shortages by assuming a constant length of replenishment cycles and a constant fraction of the shortage length with respect to the cycle. Chern et al. [17] proposed partial backlogging inventory lot-size models for deteriorating items with fluctuating demand under inflation.
Due to increasing complexities of the world economy, it is very difficult to estimate this deference precisely. Horowitz [18] discussed a simple EOQ model with a normal distribution for the inflation rate and the firm's cost of capital. Mirzazadeh and Sarfaraz [19] presented a multiple items inventory system with budget constraint and the uniform distribution for external inflation rate. Mirzazadeh [20] proposed a detailed comparison of the average annual cost and the discounted cost models under stochastic inflationary conditions. Mirzazadeh [21] developed an inventory model under stochastic inflationary conditions with variable probability density functions (pdfs) over the time horizon. He also assumed that the demand rate is dependent on the inflation rates. Ameli et al. [22] developed an inventory model to determine ordering policy for imperfect item with fuzzy defective percentage under fuzzy discounting and inflationary condition. Other considerations of inflation with nonconstant rate are Jana et al. [23], Gholami-Qadikolaei et al. [24], Neetu and Tomer [25], Mirzazadeh et al. [26], and so forth.

The demand has a considerable influence in the decisions relating to the inventory management and production planning. Although the various formations of consumption tendency have been studied, such as constant demand, pricedependent demand (e.g., [27-29]), time-dependent demand (e.g., [30-32]), and time-and-price-dependent demand (e.g., $[33,34])$, Datta and Pal [35] investigated a finite time-horizon inventory model with linear time-dependent demand rate when shortages are allowed. Here we use time-dependent rate for demand with a decreasing pattern.

$\mathrm{Wu}$ [36] investigated an inventory model with ramp type demand rate, Weibull distributed deterioration rate, and partial backlogging. Giri et al. [37] extended the ramp type demand inventory model with a more generalized Weibull deterioration.

In the literature of production planning and inventory control, most papers consider inventory systems where lead times are supposed to be equal to zero or constant. In reality, lead times are rarely constant, because unpredictable events can cause random delays. For different reasons such as machine breakdowns, transport delays, and quality problems, time of component delivery from an external supplier or processing time for the semifinished product often has an uncertain duration. Frequently, lead time fluctuations strongly degrade system performance, on the other hand, especially when we consider the effect of inflation in our inventory model. In most of the inventory models under inflation, lead times are considered to be equal to zero or constant, but we consider lead time as a fuzzy function of order quantity.

In this paper we extend the inventory models for deterioration item considering the following situations.

(i) Nonconstant deterioration rate is as follows: for the first case we consider fuzzy deterioration rate and for the second case we assume that the deterioration rate is time dependent and follows Weibull distribution with three known parameters. In most of the previous inventory models with Weibull deterioration rate, the location parameter $(\lambda)$ of Weibull distribution is considered to be equal to zero but we consider this 
parameter in our model. This means that the goods start deteriorating after elapsing a specified time $(\lambda)$.

(ii) The system is under the control of fuzzy inflation.

(iii) We introduce the fuzzy function of order quantity for lead time.

(iv) We consider a demand rate, which is a nonincreasing function of time.

The paper is organized as follows. The notation and assumptions used in the models are given in Section 2. The definitions used in the models are given in Section 3. The model with fuzzy deterioration rate is studied in Section 4.1 and the corresponding one with Weibull deterioration rate is studied in Section 4.2. The models analyses that are used to find optimum policy are given in Section 5. For each model the optimal policy is obtained. Numerical examples highlighting the results obtained are given in Section 6. The conclusion remarks are given in Section 7.

\section{Assumption and Notation}

The proposed model is developed under the following assumptions.

(1) The demand rate is a linear function of time:

$$
D(t)=a-b t \quad a>0, b>0 .
$$

(2) The replenishment rate is infinite.

(3) Shortages are allowed and fully backlogged except in the last cycle.

(4) The discount rate is $r$.

(5) The inflation rate $(\widetilde{i})$ is fuzzy in nature; therefore, the net discount rate of inflation, $(\widetilde{R})$, is also a fuzzy number, $\widetilde{R}=\tilde{i}-r$. The present worth of $p_{t}$ is $p_{t} e^{-\widetilde{R} t}, t \geq 0$ where $p_{t}$ is the value of $p$ at time $t$.

(6) Deterioration of units occurs after the specified unit of time $(\lambda)$ from the order reception moment and there is no repair or replacement of deteriorated units over the period $[0, T]$.

(7) Lead time is a function of the total ordered quantity in a period.

(8) $L_{j}$ is the lead time for period $j$ :

$$
\tilde{l_{j}}=\tilde{a} * w_{j}
$$

where $w_{j}$ is the total ordered quantity for period $j$ and $\widetilde{a}$ represents the lead time for per-unit item and it is a triangular fuzzy number $\left(a_{1}, a_{2}, a_{3}\right)$.

(9) $\theta$ is the on-hand inventory deterioration rate at time $t$ and may be a fuzzy number $(\widetilde{\theta})$ or a function of time and follows a three-parameter Weibull distribution function $\alpha \beta(t-\lambda)^{\beta-1}$.
In addition, the following are other notations that have been used in this paper:

$H$ : finite horizon time (time units),

$n$ : number of replenishment cycles during the time horizon $H$,

$T$ : length of each cycle $(T=H / n)$,

$I_{i j}(t)$ : inventory level at any time $t$ in period $j, i=1,2,3$, $j=1,2, \ldots, n$,

$C_{1}$ : holding cost per unit per unit time at time zero (\$/unit/unit time),

$C_{2}$ : shortage cost per unit per unit time at time zero (\$/unit/unit time),

$C_{3}$ : purchasing cost per unit item at time zero $(\$)$,

$\mathrm{C}_{4}$ : ordering cost per order at time zero (\$/set-up).

Additional notations will be introduced later.

\section{Definition}

Definition 1. If $X$ is a collection of objects denoted generically by $x$, then a fuzzy set $\widetilde{P}$ on $X$ is a set of order pairs.

If $x$ is continuous on $X$, then the fuzzy set is continuous and the range of $x$ is called the support of fuzzy set $P$. Continuous fuzzy sets can have various membership function shapes such as triangular and trapezoidal. In this paper, we use triangular fuzzy numbers, special case of trapezoidal, due to it straightforward structure and computational simplicity.

If $\widetilde{A}=\left(a_{1}, a_{2}, a_{3}\right)$ is a triangular fuzzy number (TFN), then $\mu_{\widetilde{A}}(x)$ is defined as follows: $\left(a_{1}, a_{2}\right.$, and $a_{3}$ are real numbers)

$$
\mu_{\widetilde{A}}(x)= \begin{cases}\frac{x-a_{1}}{a_{2}-a_{1}} & \text { for } a_{1} \leq x \leq a_{2} \\ \frac{a_{3}-x}{a_{3}-a_{2}} & \text { for } a_{2} \leq x \leq a_{3} \\ 0 & \text { otherwise. }\end{cases}
$$

Definition 2 ( $\alpha$-cut of fuzzy number). The $\alpha$-cut of a fuzzy number is a crisp set which is defined as $\widetilde{A}_{\alpha}=\{x \in R$ : $\left.\mu_{\widetilde{A}}(x) \geq \alpha\right\}$. In other words the $\alpha$-cut is a nonempty bounded closed interval, which is denoted by $\widetilde{A}_{\alpha}=\left[A_{L}(\alpha)_{\alpha}, A_{R}(\alpha)_{\alpha}\right]$.

Here $A_{L}(\alpha)_{\alpha}$ and $A_{R}(\alpha)_{\alpha}$ are the lower and upper bounds of the closed interval and

$$
\begin{aligned}
& A_{L}(\alpha)_{\alpha}=\inf \left\{x \in R: \mu_{\widetilde{A}}(x) \geq \alpha\right\}, \\
& A_{R}(\alpha)_{\alpha}=\sup \left\{x \in R: \mu_{\widetilde{A}}(x) \geq \alpha\right\} .
\end{aligned}
$$

Definition 3 (signed distance). For any $a \in R$ we define $d_{0}(a$, $0)=a$. If $a>0$ the distance from $a$ to 0 is $d_{0}(a, 0)=a$. If $a<0$, the distance from $a$ to 0 is $-d_{0}(a, 0)=-a$. This is the reason why we call $d_{0}(a, 0)$ the signed distance from $a$ to 0 . 


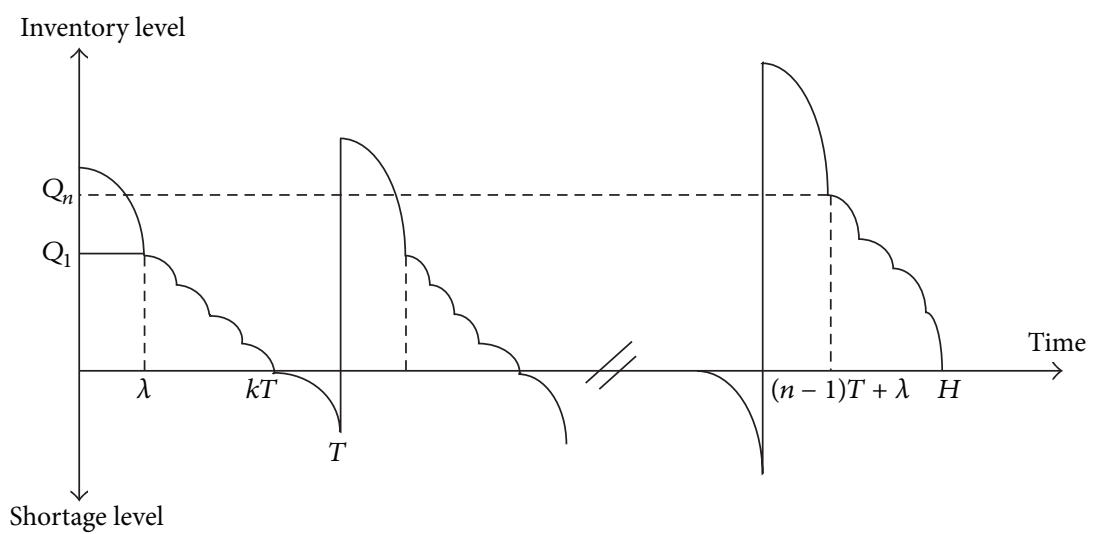

FIGURE 1: Graphical representation of the inventory system 1.

The signed distances of $A_{L}(\alpha)$ and $A_{R}(\alpha)$ to 0 are $d_{0}\left(A_{L}(\alpha), 0\right)=A_{L}(\alpha)$ and $d_{0}\left(A_{R}(\alpha), 0\right)=A_{R}(\alpha)$, respectively. We define the signed distance from 0 to the interval $\left[A_{l}(\alpha), A_{R}(\alpha)\right]$ as

$$
\begin{gathered}
d_{0}\left(\left[A_{l}(\alpha), A_{R}(\alpha)\right], 0\right) \\
=\frac{1}{2}\left(d_{0}\left(A_{L}(\alpha), 0\right), d_{0}\left(A_{R}(\alpha), 0\right)\right), \\
\frac{1}{2}\left(A_{L}(\alpha)+A_{R}(\alpha)\right)=\frac{1}{2}[a+c+(2 b-a-c) \alpha] .
\end{gathered}
$$

Since crisp interval $\left[A_{L}(\alpha), A_{R}(\alpha)\right]$ has a one-to-one correspondence with level $\alpha$ fuzzy interval $\left[A_{L}(\alpha)_{\alpha}, A_{R}(\alpha)_{\alpha}\right]$, it is natural to define the signed distance from a fuzzy interval $\left[A_{L}(\alpha)_{\alpha}, A_{R}(\alpha)_{\alpha}\right]$ to 0 as

$$
\begin{aligned}
d\left(\left[A_{L}(\alpha)_{\alpha}, A_{R}(\alpha)_{\alpha}\right], 0\right) & =d_{0}\left(\left[A_{l}(\alpha), A_{R}(\alpha)\right], 0\right) \\
& =\frac{1}{2}[a+c+(2 b-a-c) \alpha] .
\end{aligned}
$$

This is a continuous function of $\alpha$ on $0 \leq \alpha \leq 1$. We can find its average value through integration. Since $\widetilde{A}=$ $\bigcup_{0 \leq \alpha \leq 1}\left[A_{L}(\alpha)_{\alpha}, A_{R}(\alpha)_{\alpha}\right]$, we have the following definition.

Definition 4. If $\widetilde{A}=(a, b, c)$ is a triangular fuzzy number, the signed distance from $\widetilde{A}$ to $\widetilde{0}$ is defined as

$$
d(\widetilde{A}, \widetilde{0})=\int_{0}^{1} d\left(\left[A_{L}(\alpha)_{\alpha}, A_{R}(\alpha)_{\alpha}\right], 0\right) d \alpha=\frac{1}{4}(2 b+c+a) .
$$

Property 1. By applying the definition of an $\alpha$-level cut to we have the following exact arithmetic operations for continuous fuzzy set:

$$
\begin{gathered}
\widetilde{M}+\widetilde{N}=\left[m_{1}^{\alpha}, m_{2}^{\alpha}\right]+\left[n_{1}^{\alpha}, n_{2}^{\alpha}\right]=\left[m_{1}^{\alpha}+n_{1}^{\alpha}, m_{2}^{\alpha}+n_{2}^{\alpha}\right], \\
\widetilde{M}-\widetilde{N}=\left[m_{1}^{\alpha}, m_{2}^{\alpha}\right]-\left[n_{1}^{\alpha}, n_{2}^{\alpha}\right]=\left[m_{1}^{\alpha}-n_{1}^{\alpha}, m_{2}^{\alpha}-n_{2}^{\alpha}\right], \\
\widetilde{M} * \widetilde{N}=\left[m_{1}^{\alpha}, m_{2}^{\alpha}\right] *\left[n_{1}^{\alpha}, n_{2}^{\alpha}\right]=\left[m_{1}^{\alpha} * n_{1}^{\alpha}, m_{2}^{\alpha} * n_{2}^{\alpha}\right], \\
\widetilde{M} \div \widetilde{N}=\left[m_{1}^{\alpha}, m_{2}^{\alpha}\right] \div\left[n_{1}^{\alpha}, n_{2}^{\alpha}\right]=\left[m_{1}^{\alpha} \div n_{2}^{\alpha}, m_{2}^{\alpha} \div n_{1}^{\alpha}\right]
\end{gathered}
$$

\section{Model Formulation}

In the development of the model, we assume that there are $\mathrm{N}$ cycles during the real time horizon $H$. The time horizon, $H$, is divided into $n$ equal cycles each of length $T$ so that $T=H / n$.

4.1. Formulation for $j$ th $(0 \leq j \leq(n-1))$ Cycle. For Case (1), the deterioration rate is a fuzzy number.

The graphical representation of the inventory system is shown in Figure 1.

Each inventory cycle except the last cycle can be divided into three parts. Company orders an amount $I_{1 j}(0)$ of the item at time zero. Then, the level of inventory is decreasing by only the consumption rates. At the moment of $\lambda$, the item of inventory starts deteriorating. At the moment of $k T, 0 \leq$ $k \leq 1$ inventory level leads to zero and shortages occur. And at the moment $\left(K T_{j}-\widetilde{l}_{j}\right)$ of every $j$ th $(j=1,2, \ldots, n)$ cycle, company purchases an amount $Q_{j}$ units of the item. During the time interval $\left[k T_{j}, T_{j}\right]$ we do not have any deterioration, and therefore the shortages level linearly increases by the demand rate. Shortages are not allowed for the last replenishment cycle.

Because of inflation, the purchasing cost has effect on the optimal order quantity; hence, this cost has also to be included in the analysis. At the first part of $j$ th cycle there is no deterioration ( $\lambda$ unit of time after the replenishment time). The inventory level, $I_{1 j}(t)$, satisfies the following differential equations:

$$
\frac{\partial I_{1 j}(t)}{\partial t}=-a+b t \quad T(j-1) \leq t \leq T(j-1)+\lambda .
$$

The second part of the inventory level can be described as follows:

$$
\frac{\partial I_{2 j}(t)}{\partial t}=-a+b t-\tilde{\theta} I_{2 j}(t) \quad T(j-1)+\lambda \leq t \leq k T_{j} .
$$

We can solve the above differential equations by using the following equations as a boundary condition:

$$
I_{2 j}(T(j-1)+k T)=0 .
$$


We can see

$$
\begin{gathered}
I_{2 j}(t)=-\frac{b}{\theta^{2}}-\frac{a-b t}{\theta}+e^{\theta(S-t)}\left(\frac{a-b S}{\theta}+\frac{b}{\theta^{2}}\right), \\
I_{2 j}(T(j-1)+\lambda)=\frac{b}{\theta^{2}}-\frac{a+b[T(j-1)+\lambda]}{\theta} \\
+e^{\theta[T(j(k-1)+1)-\lambda]} \\
+a(T(j-1)+\lambda) \\
+\frac{1}{2} b(T(j-1)+\lambda)^{2}, \\
I_{2 j}(T(j-1)+\lambda)=I_{1 j}(T(j-1)+\lambda), \\
I_{1 j}(t)=\frac{b}{\theta^{2}}-\frac{a+b[T(j-1)+\lambda]}{\theta}+e^{\theta[T(j(k-1)+1)-\lambda]} \\
+a(T(j-1)+\lambda)+\frac{1}{2} b(T(j-1)+\lambda)^{2} \\
\quad-\left(a t+\frac{b t^{2}}{2}\right) .
\end{gathered}
$$

For the last part of $j$ th cycle, the shortages level is governed by

$$
\begin{gathered}
\frac{\partial I_{3 j}(t)}{\partial t}=-(a+b t), \quad k T_{j} \leq t \leq j T, \\
I_{3}\left(T k_{j}\right)=0, \\
I_{3 j}(t)=a k T_{j}+b k^{2} T^{2} j^{2}-\left(a t+0.5 b t^{2}\right), \quad k T_{j} \leq t \leq j T, \\
I_{31}(0)=0 .
\end{gathered}
$$

The objective of the problem is minimization of the total present value of costs over the time horizon. Consider (PVCP) as the present value of purchasing cost, (PVCH) as the present value of holding cost, (PVCS) as the present value of costs of shortages, and (PVCO) as the expected present value of cost of ordering. The total present value of costs over time horizon (TPV) is

$$
\mathrm{TPV}=\mathrm{PVCP}+\mathrm{PVCH}+\mathrm{PVCS}+\mathrm{PVCO} .
$$

4.1.1. Present Value of Holding Cost. The inventory is carried out over the first and second period of each cycle so the present value of holding cost for $j$ th cycle is given by

$$
\begin{aligned}
\operatorname{PVCH}_{j}=C_{1} & \left(\int_{T(j-1)}^{T(j-1)+\lambda} I_{1 j}(t) e^{-\widetilde{R} t} d t\right. \\
& \left.+\int_{T(j-1)+\lambda}^{T(j-1)+k T} I_{2 j}(t) e^{-\widetilde{R} t} d t\right) \\
=C_{1} & {\left[\frac{G e^{-\widetilde{R} T(j-1)}}{\widetilde{R}}\left(1-e^{-\widetilde{R} \lambda}\right)\right] }
\end{aligned}
$$

$$
\begin{aligned}
& +\frac{1}{\widetilde{R}^{3}}\left[b e^{-\widetilde{R}(T(j-1)+\lambda)}\right. \\
& \times(-\widetilde{R}(T(j-1)+\lambda) \\
& \times(2+\widetilde{R}(T(j-1)+\lambda))-2) \\
& +e^{-\widetilde{R} \lambda}((\widetilde{R}(T(j-1)) \\
& \times(2+\widetilde{R}(T(j-1)))+2))] \\
& +\frac{e^{-\widetilde{R}(T(j-1)+\lambda+K T j)}}{\widetilde{R}^{2}} \\
& *\left[e^{-\widetilde{R} K T j}(b+a \widetilde{R}+b \widetilde{R}(T(j-1)+\lambda))\right. \\
& +(b+a \widetilde{R}+b \widetilde{R} K T j)] \\
& +\frac{b}{\widetilde{R} \theta^{2}}\left[e^{-\widetilde{R}(T(j-1)+\lambda)}-e^{-\widetilde{R} K T j}\right] \\
& +e^{\theta K T j}\left[\frac{a+b K T j}{\theta}-\frac{b}{\theta^{2}}\right] \\
& *\left(\frac{e^{K T j(\widetilde{R}+\theta)}-e^{(T(j-1)+\lambda)(\widetilde{R}+\theta)}}{\widetilde{R}+\theta}\right) \text {. }
\end{aligned}
$$

So the total present value of holding cost is

$$
\mathrm{PVCH}=\sum_{j=1}^{n} \mathrm{PVCH}_{j} \text {. }
$$

4.1.2. Present Value of Shortage Cost. Shortage occurs during the third period of each cycle. The present value of the shortage cost for one cycle can be written as

$$
\begin{aligned}
\operatorname{PVCS}_{j}= & C_{2}\left(\int_{K T j}^{T j} I_{3 j}(t) e^{-\widetilde{R} t} d t\right) \\
= & \frac{a K T j+b k^{2} T^{2} j^{2}}{\widetilde{R}}\left(e^{-\widetilde{R} K T j}-e^{-\widetilde{R} T j}\right) \\
& -\frac{a e^{-\widetilde{R} T j(1+k)}}{2 R^{2}}\left(e^{-\widetilde{R} T j}(1+\widetilde{R} T j(1+k))\right. \\
& +\frac{-b e^{-\widetilde{R} T j(1+k)}}{2 \widetilde{R}} \\
& \times\left[e^{-\widetilde{R} T j}(2+\widetilde{R} K T j(2+\widetilde{R} K T j))\right. \\
& \left.-e^{-\widetilde{R} T j(1+k)}(2+\widetilde{R} T j(1+k)(2+T j(1+k)))\right] .
\end{aligned}
$$

So the total present value of shortage cost is

$$
\mathrm{PVCS}=\sum_{j=1}^{n-1} \mathrm{PVCS}_{j} \text {. }
$$




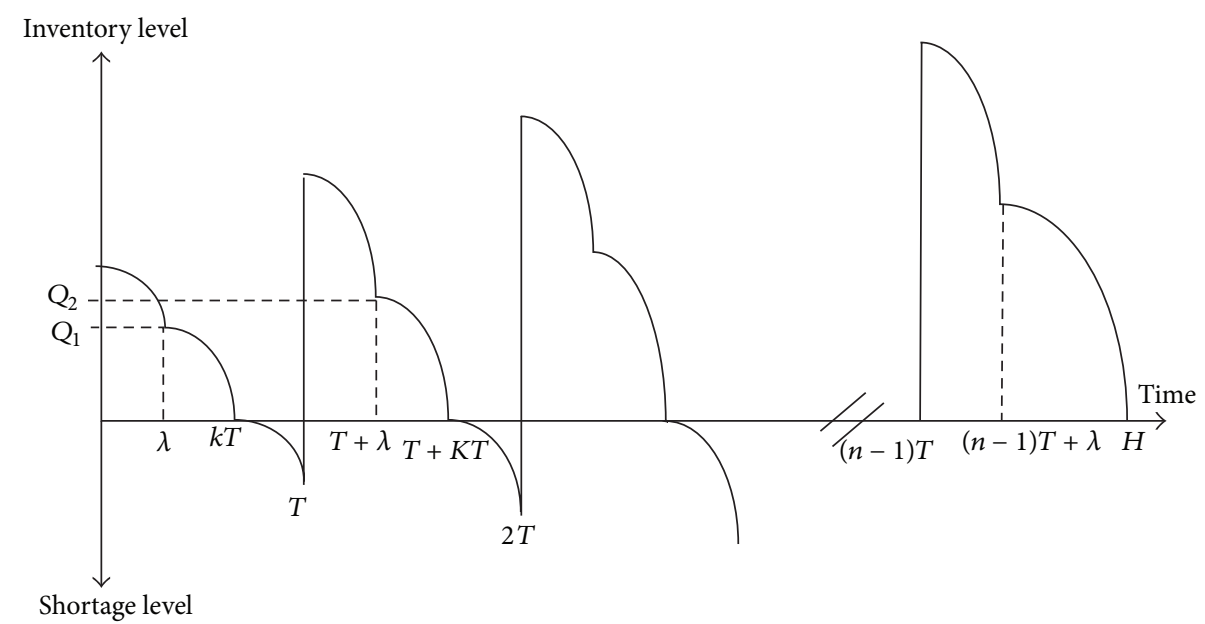

Figure 2: Graphical representation of the inventory system 2.

4.1.3. Present Value of Purchasing Cost. The ordering quantity consists of both demand and deterioration for the relevant period excluding shortage part of the period and the amount required to satisfy the demand during the shortage period in the preceding time interval. For the $j$ th cycle $(j=1,2, \ldots, n-$ 1) the present value of purchasing cost can be formulated as follows:

$$
\begin{aligned}
\operatorname{PVCP}_{j}=C_{3}( & I_{3(j-1)}(T(j-1)) \\
& \left.+I_{1 j}(T(j-1)) e^{-\widetilde{R}\left(I_{3(j-1)}(T(j-1))+I_{1 j}(T(j-1))\right)}\right) .
\end{aligned}
$$

Therefore, the total present value of purchasing cost is

$$
\begin{aligned}
\mathrm{CP}=\sum_{j=1}^{m} C_{3}( & I_{3(j-1)}(T(j-1)) \\
& \left.+I_{1 j}(T(j-1))\right) e^{-\widetilde{R}\left(I_{3(j-1)}(T(j-1))+I_{1 j}(T(j-1))\right)} .
\end{aligned}
$$

4.1.4. Present Value of Ordering Cost. Present value of sales revenue for the $j$ th $(1 \leq j \leq N)$ cycle is given by

$$
\mathrm{CA}=\sum_{j=1}^{m} \mathrm{CA}_{j}=C_{4} \sum_{j=1}^{m} e^{-\widetilde{R}\left(T j-\widetilde{l}\left(I_{3 j}(T j)+I_{2 j}(T j)\right)\right)} .
$$

4.2. Formulation for $j$ th $(0 \leq j \leq(n-1))$ Cycle. For Case (2), time-dependent (Weibull) deterioration rate, we have the following.

The graphical representation of the inventory system is shown in Figure 2.

The methodology of this case is like the previous case except in this case deterioration rate follows three-parameter Weibull distributions.
Three-parameter density function for shelf life product is defined as

$$
f(x)=\alpha \beta(t-\lambda)^{\beta-1} e^{-\alpha(t-\lambda)^{\beta}}, \quad t \geq \lambda,
$$

where $\alpha$ is the scale parameter $(\alpha>0), \beta$ is the shape parameter $(\beta>0)$, and $\lambda$ is the location parameter so the cumulative distribution function of $f(t)$ is $F(t)=e^{-\alpha t^{\beta}}$. Therefore, $R(t)=1-F(t)$ and finally Weibull instantaneous rate function for the stocked item is

$$
\theta(t)=\frac{f(t)}{1-F(t)}=\alpha \beta(t-\lambda)^{\beta-1}, \quad t \geq \lambda .
$$

In other words when the time of deterioration of an item follows this density function, this item starts deteriorating after $\lambda$ unit time after it is received to the inventory.

For this case the inventory level at the first part of $j$ th cycle satisfies the following differential equation:

$$
\begin{array}{r}
\frac{\partial I_{1 j}(t)}{\partial t}=-a+b t, \quad T(j-1) \leq t \leq T(j-1)+\lambda, \\
j=1,2, \ldots, m .
\end{array}
$$

In this case, the inventory levels at the second part of $j$ th cycle satisfy the following differential equation and the equation becomes

$$
\begin{gathered}
\frac{\partial I_{2 j}(t)}{\partial t}=-a+b t-\alpha \beta(t-\lambda)^{\beta-1} I_{2}(t), \\
T(j-1)+\lambda \leq t \leq T(j-1) K, \\
S=T(j-1)+K T, \\
I_{2 j}(S)=0, \\
I_{2 j}(t)=e^{-\alpha(t-\lambda)^{\beta}} \int_{t}^{S}(a-b u) e^{\alpha(u-\lambda)^{\beta}} d u, \\
T(j-1)+\lambda \leq t \leq T(j-1)+K T .
\end{gathered}
$$


So for the $I_{2 j}(\lambda)$ we have

$$
\begin{gathered}
I_{2 j}(\lambda)=\int_{\lambda}^{s}(a-b u)\left(\sum_{n=0}^{\infty} \frac{\left(\alpha(u-\lambda)^{\beta}\right)^{n}}{n !}\right) \\
\cong a(s-\lambda)+\frac{\alpha a(s-\lambda)^{\beta+1}}{\beta+1} \\
-b\left[\frac{(s-\lambda)^{2}}{2}+\lambda(s-\lambda)\right. \\
\left.+\alpha\left(\frac{(s-\lambda)^{\beta+2}}{\beta+2}+\lambda \frac{(s-\lambda)^{\beta+1}}{\beta+1}\right)\right]=Q_{j}, \\
I_{1 j}(\lambda)=I_{2 j}(\lambda)=Q_{j}, \\
I_{1 j}(t)=\frac{b t^{2}}{2}-a t+Q_{j}-\frac{b \lambda^{2}}{2}+a \lambda, \\
T(j-1) \leq t \leq T(j-1)+\lambda .
\end{gathered}
$$

For the last part of $j$ th cycle, the shortages level formulation is similar to the first case. The detailed analysis is given as follows.

4.2.1. Present Value of Holding Cost. The present value of holding cost for $j$ th cycle is given by

$$
\begin{gathered}
C H_{j}=C_{1}\left(\int_{T(j-1)}^{T(j-1)+\lambda} I_{1 j}(t) e^{-\widetilde{R} t} d t+\int_{T(j-1)+\lambda}^{K T j} I_{2 j}(t) e^{-\widetilde{R} t} d t\right) \\
=C_{1}\left(\left[\frac{G e^{-\widetilde{R} T(j-1)}}{\widetilde{R}}\left(1-e^{-\widetilde{R} \lambda}\right)\right]\right. \\
+\frac{1}{\widetilde{R}^{3}}\left[b e^{-\widetilde{R}(T(j-1)+\lambda)}\right. \\
\times(-\widetilde{R}(T(j-1)+\lambda) \\
\times(2+\widetilde{R}(T(j-1)+\lambda))-2) \\
+e^{-\widetilde{R} \lambda}((\widetilde{R}(T(j-1)) \\
+\int_{T(j-1)+\lambda}^{S}\left(e^{-\alpha(t-\lambda)^{\beta} \int_{t}^{S}(a-b u)}\right. \\
\times(2+\widetilde{R}(T(j-1)))+2))] \\
\left.\left.\times e^{\alpha(u-\lambda)^{\beta}} d u\right) e^{-\widetilde{R} t} d t\right) .
\end{gathered}
$$

For the simplified formulation we define

$$
C=a s+\frac{a \alpha(s-\lambda)^{\beta+1}}{\beta+1}-\frac{b}{2} S^{2}-\frac{b \alpha(s-\lambda)^{\beta+1}(s b+s-\lambda)}{(\beta+2)(\beta+1)} .
$$

Therefore we have

$$
\begin{aligned}
& \mathrm{CH}_{j} \\
& \cong C_{1}\left(\left[\frac{G e^{-\widetilde{R} T(j-1)}}{\widetilde{R}}\left(1-e^{-\widetilde{R} \lambda}\right)\right]+\frac{1}{\widetilde{R}^{3}}\right. \\
& \times\left[b e^{-\widetilde{R}(T(j-1)+\lambda)}\right. \\
& \times(-\widetilde{R}(T(j-1)+\lambda)(2+\widetilde{R}(T(j-1)+\lambda))-2) \\
& \left.+e^{-\widetilde{R} \lambda}((\widetilde{R}(T(j-1))(2+\widetilde{R}(T(j-1)))+2))\right] \\
& +C(K T-\lambda)-\frac{(R C+a)}{2}\left[S^{2}-T(j-1)+\lambda^{2}\right] \\
& -\frac{a \alpha\left[(T(j-1)+\lambda)^{\beta+2}-(T(j-1))^{\beta+2}\right]}{(\beta+1)(\beta+2)} \\
& +\frac{(b+2 R a)\left[S^{3}-(T(j-1)+\lambda)^{3}\right]}{6} \\
& +\left(b \alpha \left[(T(j-1)+\lambda)^{\beta+2}\right.\right. \\
& \times\left(S\left(2+3 \beta+\beta^{2}\right)-2 \lambda-T(j-1)^{\beta+2}\right. \\
& \times((T(j-1)+\lambda) \\
& \left.\left.\left.\left.\times\left(2+3 \beta+\beta^{2}\right)-2 \lambda\right)\right)\right]\right) \\
& \times\left((\beta+1)(\beta+3)(\beta+2)^{2}\right)^{-1} \\
& +\left(a ^ { 2 } \left[(T(j-1)+\lambda)^{\beta+1}\right.\right. \\
& \times(S \beta+\lambda+K T j)-T(j-1)^{\beta+1} \\
& \times((T(j-1)+\lambda)(\beta)+2 \lambda+T(j-1))]) \\
& \times((\beta+1)(\beta+2))^{-1} \\
& +\frac{\alpha a^{2}\left[(S-\lambda)^{2(\beta+1)}-(T(j-1))^{2(\beta+1)}\right]}{2(\beta+1)^{2}} \\
& -\frac{b a}{2(\beta+1)(\beta+3)(\beta+2)} \\
& \times\left[(s-\lambda)^{\beta+1}\right. \\
& \times\left(2 \lambda S(\beta+1)+K^{2} T^{2} j^{2} \beta(\beta+3)\right. \\
& \left.+2 \lambda^{2}+2 K^{2} T^{2} j^{2}\right) \\
& -(T(j-1))^{\beta+1} \\
& \times\left(2 \lambda(T(j-1)+\lambda)(\beta+1)+F^{2} \beta(\beta+3)\right. \\
& \left.\left.+2 \lambda^{2}+2(T(j-1)+\lambda)^{2}\right)\right]
\end{aligned}
$$




$$
\begin{aligned}
& -\frac{a c\left[(S-\lambda)^{\beta+1}-(T(j-1))^{\beta+1}\right]}{\beta+1} \\
& +\left(\operatorname { R a \alpha } \left[(S-\lambda)^{\beta+2}(S(\beta+2)+\lambda)-(T(j-1))^{\beta+2}\right.\right. \\
& \quad \times((T(j-1)+\lambda)(\beta+2)+\lambda)]) \\
& \times((\beta+1)(\beta+3)(\beta+2))^{-1} \\
& \left.-\frac{R b\left[K^{4} T^{4} j^{4}-(T(j-1)+\lambda)^{4}\right]}{8}\right) .
\end{aligned}
$$

Present Value of Shortage Cost. The present value of shortage cost is similar to the previous case.

4.2.2. The Present Value of Purchasing Cost. The total amount that we have to purchase for $j$ th cycle is

$$
I_{1(j)}(T j)+I_{3(j-1)}(T j) .
$$

The lead time for this amount of item is $l_{j}=\widetilde{l} w_{j}$.

So the total present value of purchasing cost is

$$
\begin{aligned}
\text { PVCP }= & C_{3} I_{11}(0) \\
& +C_{3} \sum_{j=1}^{n-1}\left(I_{1(j)}(T j)-I_{3(j-1)}(T j)\right) \\
& \times e^{-\widetilde{R}\left(T j-\widetilde{l}\left(\left(I_{1(j)}\right)(T j)-I_{3(j-1)}(T j)\right)\right)} .
\end{aligned}
$$

4.2.3. The Present Value of Ordering Cost. For the $j$ th cycle we have to order at $T(j-1)-\widetilde{l}\left(I_{3(j-1)}(T(j-1))+I_{1 j}(T(j-1))\right)$.

So the total present value of ordering cost for the $j$ th cycle is

$$
\mathrm{PVCO}=\sum_{j=1}^{m} C A_{j}=C_{4} \sum_{j=1}^{m} e^{-\widetilde{R}\left(T j-\tilde{l}\left(I_{3 j}(T j)+I_{2 j}(T j)\right)\right)} .
$$

4.2.4. Present Value of Deteriorated Item Cost. The amount of deteriorated items during $[T(j-1)+\lambda, T(j-1)+k T]$ is

$$
\begin{aligned}
I_{2 j}(\lambda)-\int_{\lambda}^{S} D(t) d t= & a(s-\lambda)+\frac{\alpha a(s-\lambda)^{\beta+1}}{\beta+1} \\
- & b\left[\frac{(s-\lambda)^{2}}{2}+\lambda(s-\lambda)\right. \\
& \left.+\alpha\left(\frac{(s-\lambda)^{\beta+2}}{\beta+2}+\lambda \frac{(s-\lambda)^{\beta+1}}{\beta+1}\right)\right] \\
& -a(K T j-\lambda)+\frac{b\left(k^{2} T^{2} j^{2}-\lambda^{2}\right)}{2} .
\end{aligned}
$$

The total present value of the amount of deteriorated items cost is

PVCD

$$
\begin{aligned}
& =\sum_{j=1}^{m} C D_{j} \\
& =\widetilde{\theta} C_{5} \sum_{j=1}^{m} \int_{T(j-1)+\lambda}^{K T j}\left(e^{-\alpha(t-\lambda)^{\beta}} \int_{t}^{S}(a-b u) e^{\alpha(u-\lambda)^{\beta}} d u\right) e^{-\widetilde{R} t} d t .
\end{aligned}
$$

The total present value of costs over time horizon in this case (TPV) is

$$
\mathrm{TPV}=\mathrm{PVCP}+\mathrm{PVCH}+\mathrm{PVCS}+\mathrm{PVCO}+\mathrm{PVCD} .
$$

\section{Model Analysis}

The problem is to determine the optimal values of $n$ and $k$ so as to minimize the total present value of inventory system costs:

$$
\mathrm{TPV}=\mathrm{PVCP}+\mathrm{PVCH}+\mathrm{PVCS}+\mathrm{PVCO}+\mathrm{PVCD} .
$$

For this, the algorithm begins by setting discrete variable $n=1$ and takes the derivatives of $\operatorname{TPV}(n, k)$ with respect to $k$. Equating the derivative to zero derives the following necessary conditions of optimality $\partial T P V / \partial k=0$.

For a given value of $n$ derive $k^{*}$ from the above equation $\operatorname{TPV}\left(n, k^{*}\right)$ is derived by substituting $\left(n, k^{*}\right)$ into (18). Then, $n$ increases by increment of one continually and TPV $\left(n, k^{*}\right)$ is driven again. The above stages repeat until the minimum $\operatorname{TPV}\left(n, k^{*}\right)$ can be found.

Since the inflation rate, deterioration rate, and the lead time are fuzzy numbers the objective function becomes fuzzy. We first use the singed distance method to defuzzify the objective function. Then since the derived function is complicated we use the pervious algorithm for optimizations system.

\section{Numerical Example}

The numerical example is provided to clarify how the proposed model is applied (see Table 1).

For the first case the result of the numerical example is shown in Table 2.

For the second case the result of the numerical example is shown in Table 3.

\section{Conclusion}

In this paper, an inventory model with time-dependent demand studied under two different deterioration rates: (a) time-dependent (Weibull) deterioration rate and (b) fuzzy deterioration rate. The items are not deteriorating as soon as they received to the inventory but they start to deteriorate after a specified interval. The lead time is a function of 
TABLE 1: The parameter values for numerical example.

\begin{tabular}{lc}
\hline$C_{1}$ & 0.5 \\
$C_{2}$ & 3.5 \\
$C_{3}$ & 3 \\
$C_{4}$ & 50 \\
$\widetilde{l}$ & $(0.000434,0.000651,0.000868)$ \\
$\widetilde{\theta}$ & $(0.086,0.11,0.205)$ \\
$\widetilde{R}$ & $0.1,0.15)$ \\
$\alpha$ & 3 \\
$\beta$ & 0.028 \\
$\lambda$
\end{tabular}

TABLE 2: Data for numerical example (Case 1).

\begin{tabular}{lcc}
\hline$N$ & $k$ & TPV \\
\hline 1 & 0.30065 & 113917.42 \\
$2^{*}$ & $0.29804^{*}$ & $43788.11^{*}$ \\
3 & 0.29315 & 48725.02 \\
4 & 0.29307 & 55435.08 \\
5 & 0.29104 & 90183.22 \\
\hline
\end{tabular}

TABLE 3: Data for numerical example (Case 2).

\begin{tabular}{lcc}
\hline$N$ & $k$ & TPV \\
\hline $1^{*}$ & $0.2054^{*}$ & $4.6 \times 10^{8^{*}}$ \\
2 & 0.2448 & $6.8 \times 10^{8}$ \\
3 & 0.27315 & $7.2 \times 10^{8}$ \\
4 & 0.31023 & $9.3 \times 10^{8}$ \\
5 & 0.35002 & $1.2 \times 10^{9}$ \\
\hline
\end{tabular}

the amount that we ordered; we consider both inflation rate and the lead time for preparing one item as fuzzy parameter. Total present costs of inventory system were minimized and numerical examples were proposed to illustrate findings. Different from the previous studies here we are able to consider the rate of deterioration as two kinds beside each other and compare the solutions of these two cases with each other when all of the other parameters are the same. The results show that the total cost in the second case (considering stochastic deterioration) is more than the first case (considering fuzzy deterioration).

\section{Conflict of Interests}

The authors declare that there is no conflict of interests regarding the publication of this paper.

\section{References}

[1] P. M. Ghare and G. H. Schrader, "A model for exponentially decaying inventory system," International Journal of Production Research, vol. 21, pp. 449-460, 1963.

[2] R. P. Covert and G. C. Philip, "An EOQ model for items with Weibull distribution deterioration," AIIE Trans, vol. 5, no. 4, pp. 323-326, 1973.
[3] I. Moon, B. C. Giri, and B. Ko, "Economic order quantity models for ameliorating/deteriorating items under inflation and time discounting," European Journal of Operational Research, vol.162, no. 3, pp. 773-785, 2005.

[4] J.-M. Chen, "An inventory model for deteriorating items with time-proportional demand and shortages under inflation and time discounting," International Journal of Production Economics, vol. 55, no. 1, pp. 21-30, 1998.

[5] S. S. Sana, "The stochastic EOQ model with random sales price," Applied Mathematics and Computation, vol. 218, no. 2, pp. 239248, 2011.

[6] S. S. Sana, "Optimal selling price and lotsize with time varying deterioration and partial backlogging," Applied Mathematics and Computation, vol. 217, no. 1, pp. 185-194, 2010.

[7] Z. T. Balkhi, "An optimal solution of a general lot size inventory model with deteriorated and imperfect products, taking into account inflation and time value of money," International Journal of Systems Science, vol. 35, no. 2, pp. 87-96, 2004.

[8] S. K. De and S. S. Sana, "Fuzzy order quantity inventory model with fuzzy shortage quantity and fuzzy promotional index," Economic Modelling, vol. 31, no. 1, pp. 351-358, 2013.

[9] S.-T. Lo, H.-M. Wee, and W.-C. Huang, "An integrated production-inventory model with imperfect production processes and Weibull distribution deterioration under inflation," International Journal of Production Economics, vol. 106, no. 1, pp. 248-260, 2007.

[10] S. S. Sana, "The EOQ model-a dynamical system," Applied Mathematics and Computation, vol. 218, no. 17, pp. 8736-8749, 2012.

[11] R. Roy Chowdhury, S. K. Ghosh, and K. S. Chaudhuri, "An inventory model for perishable items with stock and advertisement sensitive demand," International Journal of Management Science and Engineering Management, vol. 9, no. 3, pp. 169-177, 2014.

[12] T. Singh and H. Pattnayak, "Two-warehouse inventory model for deteriorating items with linear demand under conditionally permissible delay in payment," International Journal of Management Science and Engineering Management, vol. 9, no. 2, pp. 104113, 2014.

[13] C. K. Sivashankari and S. Panayappan, "Production inventory model with reworking of imperfect production, scrap and shortages," International Journal of Management Science and Engineering Management, vol. 9, no. 1, pp. 9-20, 2014.

[14] J. A. Buzacott, "Economic order quantity with inflation," Operational Research Quarterly, vol. 26, no. 3, pp. 553-558, 1975.

[15] M. Soleimani-Amiri, S. Nodoust, A. Mirzazadeh, and M. Mohammadi, "The average annual cost and discounted cost mathematical modeling methods in the inflationary inventory systems-a comparison analysis," SOP Transactions on Applied Mathematics, vol. 1, no. 1, pp. 31-41, 2014.

[16] K. Hou, "An inventory model for deteriorating items with stockdependent consumption rate and shortages under inflation and time discounting," European Journal of Operational Research, vol. 168, no. 2, pp. 463-474, 2006.

[17] M. Chern, H. Yang, and J. L. Teng, "Partial backlogging inventory lot-size models for deteriorating items with fluctuating demand under inflation," European Journal of Operational Research, vol. 191, no. 1, pp. 127-141, 2008.

[18] I. Horowitz, "EOQ and inflation uncertainty," International Journal of Production Economics, vol. 65, no. 2, pp. 217-224, 2000 . 
[19] A. Mirzazadeh and A. R. Sarfaraz, "Constrained multiple items optimal order policy under stochastic inflationary conditions," in Proceedings of the 2nd Annual International Conference on Industrial Engineering Application and Practice, pp. 725-730, San Diego, Calif, USA, 1997.

[20] A. Mirzazadeh, "A comparison of the mathematical modeling methods in the inventory systems under uncertain conditions," International Journal of Engineering Science and Technology, vol. 3, pp. 6131-6142, 2011.

[21] A. Mirzazadeh, "Inventory management under stochastic conditions with multiple objectives," Artificial Intelligence Research, vol. 2, pp. 16-26, 2013.

[22] M. Ameli, A. Mirzazadeh, and M. A. Shirazi, "Economic order quantity model with imperfect items under fuzzy inflationary conditions," Trends in Applied Sciences Research, vol. 6, no. 3, pp. 294-303, 2011.

[23] D. K. Jana, B. Das, and T. K. Roy, "A partial backlogging inventory model for deteriorating item under fuzzy inflation and discounting over random planning horizon: a fuzzy genetic algorithm approach," Advances in Operations Research, vol. 2013, Article ID 973125, 13 pages, 2013.

[24] A. Gholami-Qadikolaei, A. Mirzazadeh, and R. TavakkoliMoghaddam, "A stochastic multiobjective multiconstraint inventory model under inflationary condition and different inspection scenarios," Journal of Engineering Manufacture, vol. 227, no. 7, pp. 1057-1074, 2013.

[25] A. K. Neetu and A. Tomer, "Deteriorating inventory model under variable inflation when supplier credits linked to order quantity," Procedia Engineering, vol. 38, pp. 1241-1263, 2012.

[26] A. Mirzazadeh, M. M. Seyyed Esfahani, and S. M. T. Fatemi Ghomi, "An inventory model under certain inflationary conditions, finite production rate and inflation-dependent demand rate for deteriorating items with shortages," International Journal of Systems Science, vol. 40, no. 1, pp. 21-31, 2009.

[27] P. L. Abad, "Optimal price and order size for a reseller under partial backordering," Computers and Operations Research, vol. 28, no. 1, pp. 53-65, 2001.

[28] P. L. Abad, "Optimal pricing and lot-sizing under conditions of perishability and partial backordering," Management Science, vol. 42, no. 8, pp. 1093-1104, 1996.

[29] K. L. Hou and L. C. Lin, "Optimal pricing and ordering policies for deteriorating items with multivariate demand under trade credit and inflation," OPSEARCH, vol. 50, no. 3, pp. 404-417, 2013.

[30] B. Sarkar, S. S. Sana, and K. Chaudhuri, "An imperfect production process for time varying demand with inflation and time value of money: an EMQ model," Expert Systems with Applications, vol. 38, no. 11, pp. 13543-13548, 2011.

[31] S. M. Mousavi, V. Hajipour, S. T. Niaki, and N. Alikar, "Optimizing multi-item multi-period inventory control system with discounted cash flow and inflation: two calibrated meta-heuristic algorithms," Applied Mathematical Modelling, vol. 37, no. 4, pp. 2241-2256, 2013.

[32] S. R. Singh, V. Gupta, and P. Gupta, “Three stage supply chain model with two warehouse, imperfect production, variable demand rate and inflation," International Journal of Industrial Engineering Computations, vol. 4, no. 1, pp. 81-92, 2013.

[33] M. Ghoreishi, A. Arshsadi Khamseh, and A. Mirzazadeh, "Joint optimal pricing and inventory control for deteriorating items under inflation and customer returns," Journal of Industrial Engineering, vol. 2013, Article ID 709083, 7 pages, 2013.
[34] T. Hsieh and C. Y. Dye, "Pricing and lot-sizing policies for deteriorating items with partial backlogging under inflation," Expert Systems with Applications, vol. 37, no. 10, pp. 7234-7242, 2010.

[35] T. K. Datta and A. K. Pal, "Effects of inflation and time-value of money on an inventory model with linear time-dependent demand rate and shortages," European Journal of Operational Research, vol. 52, no. 3, pp. 326-333, 1991.

[36] K. S. Wu, "An EOQ inventory model for items with Weibull distribution deterioration, ramp type demand rate and partial backlogging," Production Planning and Control, vol. 12, no. 8, pp. 787-793, 2001.

[37] B. C. Giri, A. K. Jalan, and K. S. Chaudhuri, "Economic order quantity model with Weibull deterioration distribution, shortage and ramp-type demand," International Journal of Systems Science, vol. 34, no. 4, pp. 237-243, 2003. 

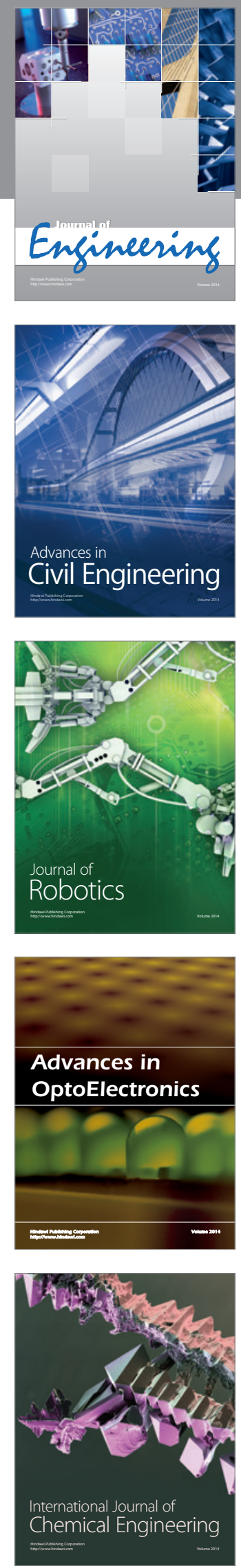

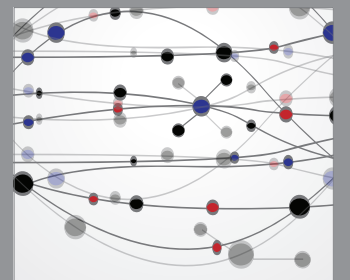

The Scientific World Journal
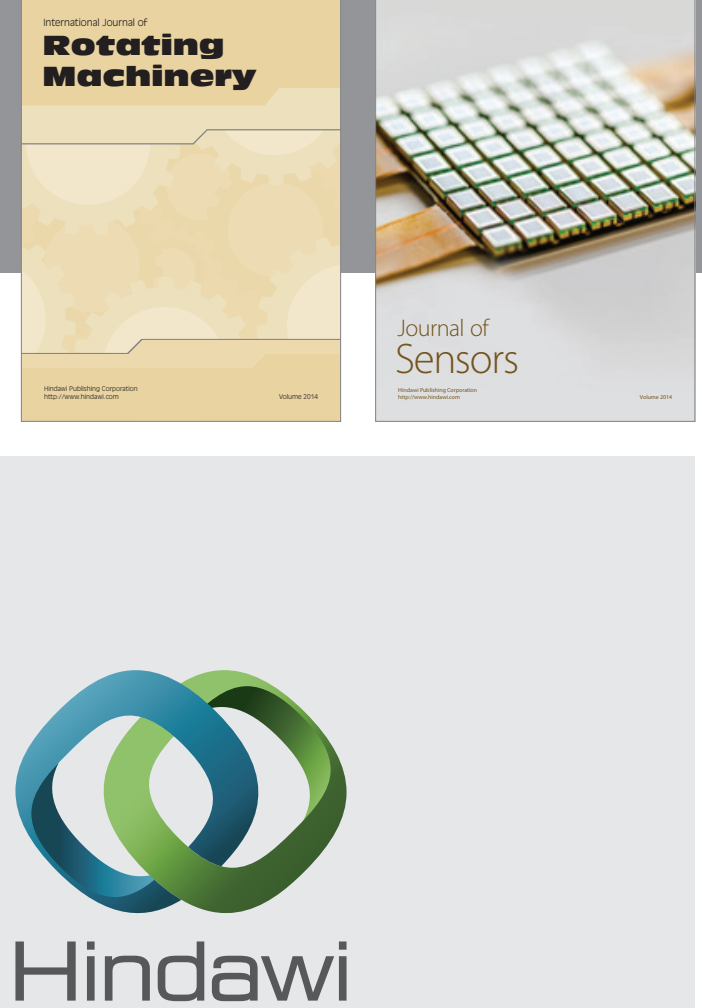

Submit your manuscripts at http://www.hindawi.com
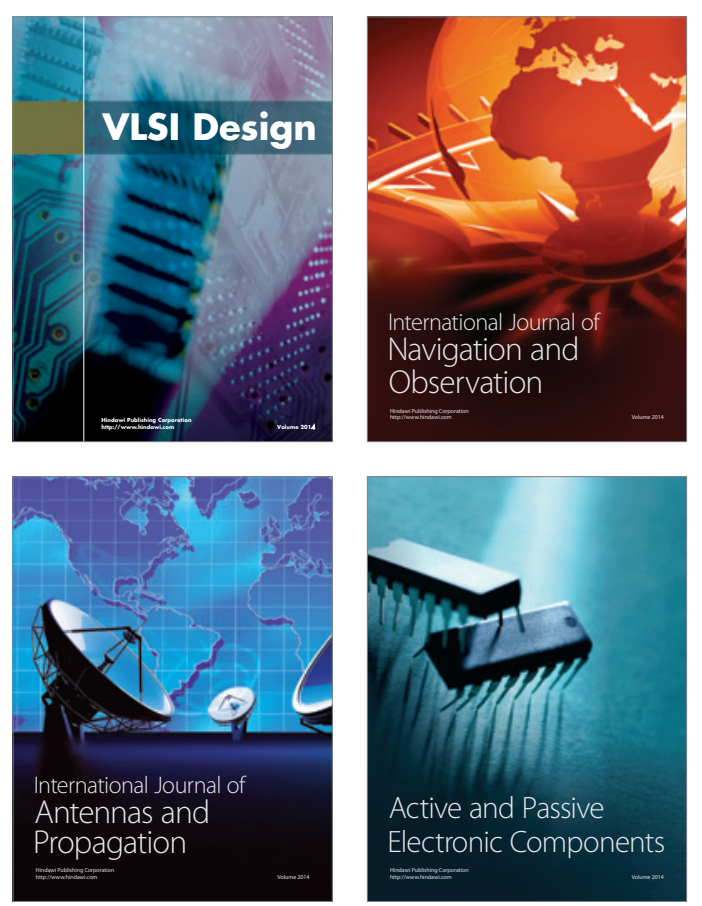
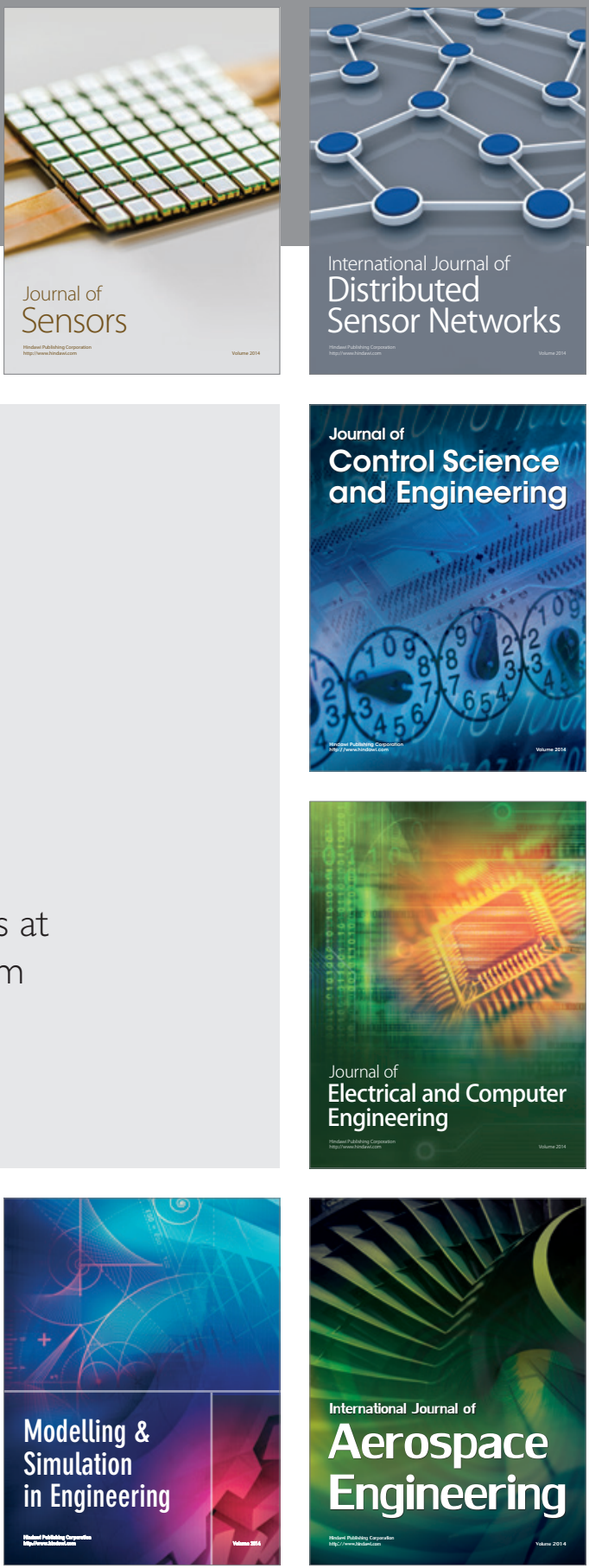

Journal of

Control Science

and Engineering
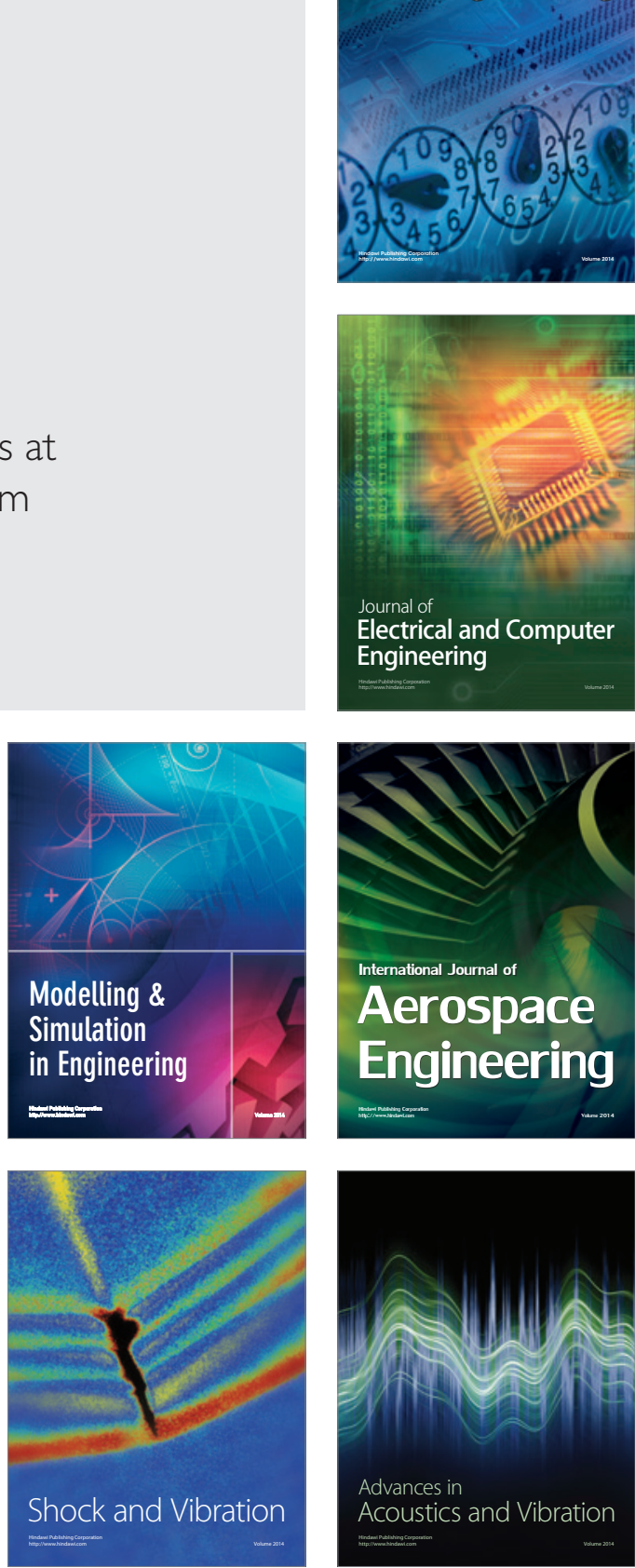